\title{
Operating Radio Controlling Car via the Internet Network
}

\author{
Chomtip Pornpanomchai, Member, IACSIT, and Nutdanai Trakarnsirinont
}

\begin{abstract}
The objective of this research is to operate a radio-controlled car ( $\mathrm{RC}$ car) via the Internet network, which is called "Tele RC Car or (TRCC)". The research was conducted by taking user commands from a client computer machine, which is connected to a server computer via the Internet network. The server computer connects with a digital-to-radio signal converter. Finally, an RC car operated based on the radio wave commands, which is received from the client computer. Both client and server computers were installed at different room in the same building. The experiment was conducted on the RC car with nine basic commands and three pattern commands. The nine basic commands consist of: 1) stop, 2) forward, 3) reverse, 4) turn left, 5) turn right, 6) forward \& turn left, 7) reverse \& turn left, 8) forward \& turn right, and 9) reverse $\&$ turn right. The three pattern commands consist of 1 ) the $O$-shape pattern, 2) the $\infty$-shape pattern and 3) to run $\&$ stop before hit an object. The experimental precisions are 100 percent for basic commands and 93 percent for pattern commands.
\end{abstract}

Index Terms-Radio controller car, Digital-to-radio signal converter, Client-server computers, Internet network.

\section{INTRODUCTION}

In the era of technology, there are two great human innovations, which are computer technology and robotic technology. We use a computer system to perform various difficult tasks, namely: 1) chromosome matching, 2) Internet communication, 3) e-commerce, and 4) robotic controlling etc. Based on the usefulness of computers, it is very difficult to imagine, how people will live without a computer system.

The second great human innovation is a robotic system. There are many kinds of robots with such tasks as: 1) a robot arm to service an industrial purpose, 2) an army robot to detonate a buried bomb, 3) an android robot to do a human job, 4) a mobile robot to move around the environment like an automatic guided vehicle (AGV), and 5) children's toy car or an RC car that can be operated with an radio wave, etc. Normally, a robotic system needs some computer systems to manage it. The complexity of computer software depends on how difficult robotic tasks are. In the near future, we hope that we can integrate a computer with a robotic system to build a sophisticated machine, for example, an unmanned driving car.

This research uses a client computer to control an RC car, which is connected with a server computer via the Internet

Manuscript received October 3, 2011; revised December 11, 2011.

Dr. Chomtip Pornpanomchai is with the Faculty of Information and Communication Technology, Mahidol University, Bangkok 10400, Thailand, (e-mail: itcpp@mahidol.ac.th)

Nutdanai Trakarnsirinont is a graduate student in the Faculty of Information and Communication Technology, Mahidol University, Bangkok 10400,Thailand(e-mail:(u4988225,u4988055,u4988023)@student.mahidol. ac.th). network. The experiments were conducted on two types of commands, namely: basic commands and pattern commands. The basic commands are simple RC car controller commands e.g. forward, reverse, turn left, turn right and stop etc. The pattern commands consist of three specific commands, which are 1) run as the O-shape, 2) run as the $\infty$-shape and 3) run \& stop before hit an object. Normally, the RC car has a limitation to control a car via radio signal in a long distance. Therefore to extend an RC car operation, all the experiments in this research try to prove that the RC car can operate by client-server computers.

\section{LITERATURE REVIEWS}

In this section, we discuss research works related to the remote RC-car via the Internet network that describe in the previous section. Section II.A describes the researches on controlling an $\mathrm{RC}$ car by a computer and Section II.B explains the researches on controlling robot via the Internet network. Both researches have the following details.

\section{A. Controlling an RC Car by a Computer}

There is a group of researchers who develop an RC car controlled by a computer and apply it in many applications, which have the following briefly details:

Chomtip P. et al. controlled an RC car by using both voice and facial commands. The research used a computer to convert digital signal to radio signal, then controlled RC car based on the radio signal command. [1]-[3]

Masanori Y. et al. used four RC cars to play a mini-car-soccer game. They captured competition pictures by a video camera on the ceiling and used video pictures to operate the soccer game. [4]

Christophe C. and Pierre B. applied a mobile robot for chasing an elusive target in an unknown and unconstrained environment. [5]

Yeung Y. and Kai M. T. scaled down a driver-tested performance by using an $\mathrm{RC}$ car as the output of the evaluation. [6]

Kenichi A. et al. controlled an $\mathrm{RC}$ car via the internet network by putting the computer server on the second floor and using a client to manage the $\mathrm{RC}$ car on the eighth floor of the same building. The experiment conducted on some regulation and simple line tracking controls and a small radius circle. [7]

\section{B. Controlling Robot Via the Internet Network}

There are many researchers controlling robot via the Internet network, which have the following briefly details:

Chomtip P. and Phichate S. presented robot arm controlling via the TCP/IP network with many operation scenarios. [8]

Jae-won K. et al. demonstrated how to control robot 
movement by the MPEG-4 video and the G723 audio via the Internet network using WLAN. [9]

Dawei W. et al. presented the Internet-based teleoperation system with an omni-directional mobile robot. A remote user can used client computer and remote live video to control the robot via the Internet network. [10]

Riko S. et al. described a method of education and training the trainee. When tasks are satisfied of the trainee, then, they exported to remote a robot arm via the Internet for a real-world execution. [11]

Shuang C. and Junnan W. conducted a robot arm teleoperation system based on the Internet network. They used tele-robot-arm to draw a picture, which looks like four petals flower. [12]

Marin R. et al. presented system architecture of the Internet based tele-laboratory, which allows users to remote control and program online robot. [13]

Based on the researches, this research objective is to purpose the controlling RC car via the Internet network. The details of the research are presented in the methodology section.

\section{METHODS}

This section introduces an approach to create the TRCC system. First, the conceptual diagram is presented and then a transformation of the conceptual diagram into the system structure chart is presented. Finally, each component of the structure chart is described for more details.

\section{A. Conceptual Diagram}

According to our study, we design a conceptual diagram of the TRCC system, as shown in Fig. 1. The research is separated into two parts. The first part is the client part for a user to send the remote commands to operate the $\mathrm{RC}$ car. The second part is the server part for receiving remote command and operating the $\mathrm{RC}$ car based on the received operation. Both server and client system are installed in the Internet network for a remote controlling purpose.

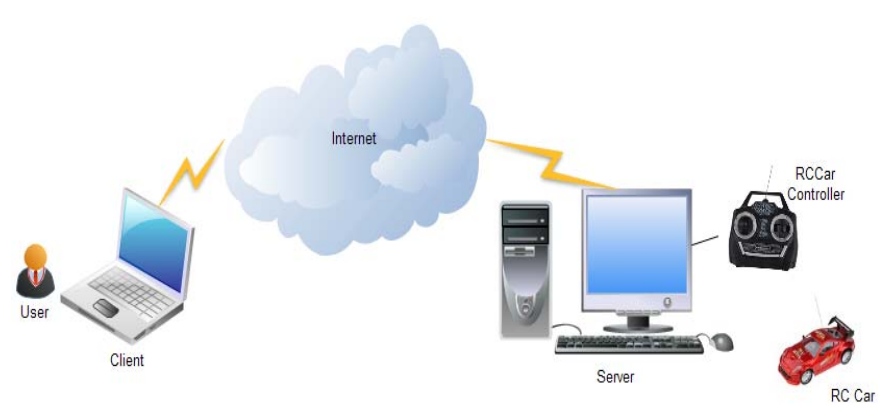

Fig. 1. The conceptual diagram of the TRCC system.

\section{B. System Structure Chart}

We have converted the TRCC conceptual diagram in the previous section into a structure chart as shown in Fig. 2. In the TRCC structure chart, it consists of three main modules of: 1) Network connection, 2) Get user commands and 3) RC car controller, the details of each module have the following details.

\section{1) Network connection}

This module connects a client computer to a server computer via the Internet network by using the TCP/IP protocol. The TRCC system uses port number 8080 to transfer the data between the client and server computers. This module consists of 2 sub-modules, which are the client connection sub-module and the server connection sub-module. Both sub-modules operate the TCP/IP connection between client-server computers.

2) Get user commands

This module allows user to sent commands to control RC car via the Internet. There are 2 types of commands, which are the basic commands and the pattern commands.

The basic commands are simple RC car operations, e.g. 1) stop, 2) forward, 3) reverse, 4) turn left, 5) turn right, 6) forward \& turn left, 7) reverse \& turn left, 8) forward \& turn right, and 9) reverse \& turn right. The basic commands user interface screen is shown in Fig. 3, which consists of a communication TCP/IP address box (label number 1), a communication port box (label number 2) and connection button (label number 3). The nine basic command buttons locate below the connection button, which are: 1) Forward + Turn Left button (label number 4), 2) Forward button (label number 5), 3) Forward + Turn Right button (label number 6), 4) Left button (label number 7), 5) Stop button (label number 8), 9) Right button (label number 9), 10) Reverse + Turn Left button (label number 10), 10) Reverse (label number 11), 10) Reverse and Turn Right button (label number 12).

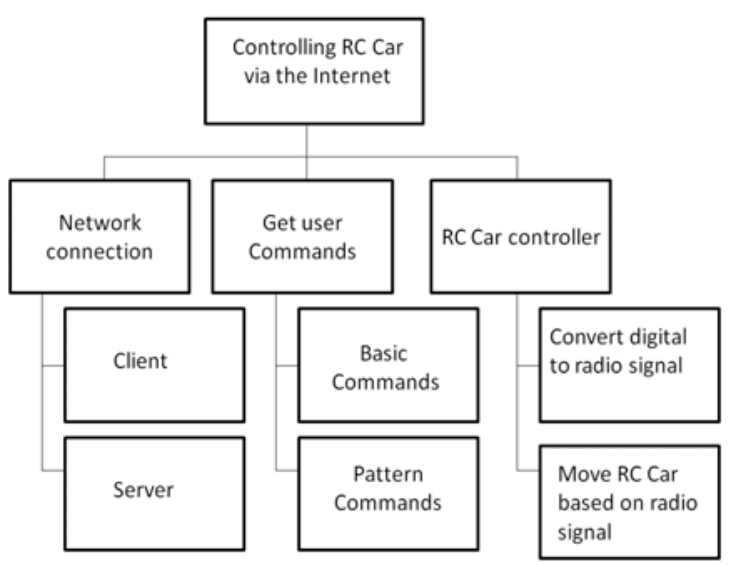

Fig. 2. The TRCC system structure chart.

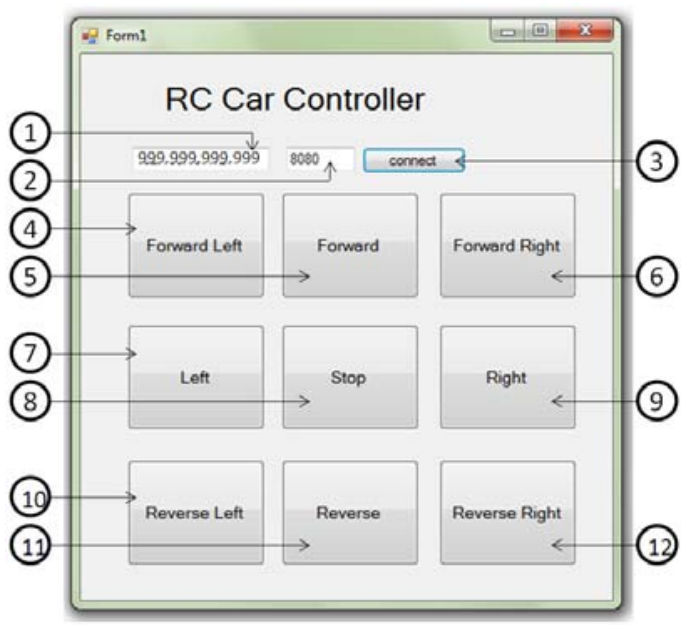

Fig. 3. The basic commands user interface screen.

The pattern commands are specific commands for operate $\mathrm{RC}$ car, namely: 1) run as the O-shape, 2) run as the $\infty$-shape and 3) run \& stop before hit an object. The pattern commands 
user interface screen is shown in Fig. 4, which consists of a communication TCP/IP address box (label number 1), a communication port box (label number 2) and connection button (label number 3 ). The three pattern command buttons locate below connection button, which are: 1) the infinity shape button (label number 4), 2) the circle shape button (label number 5), the stop before hit an object button (label number 6), and the stop button (label number 7).

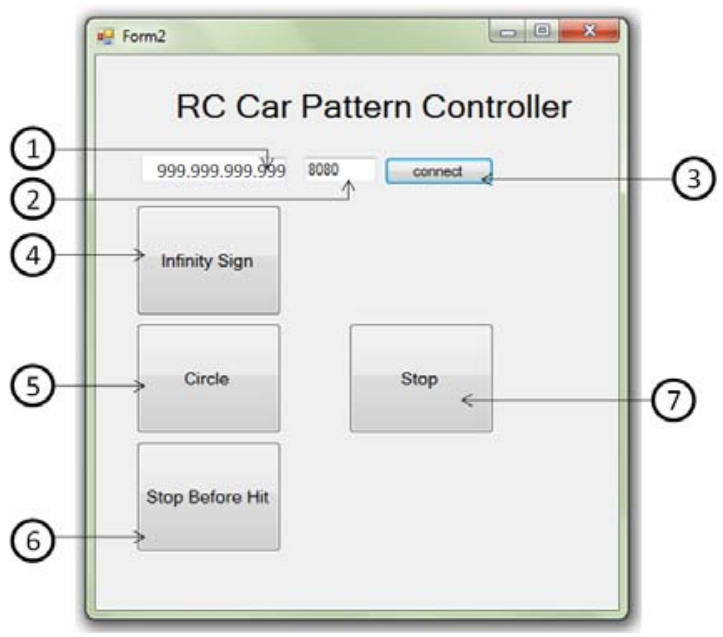

Fig. 4. The pattern commands user interface screen.

\section{1) RC car controller}

This module converts a client RC car command into a digital signal, after that the TRCC system converts a digital signal into a radio signal. Finally, the RC car moves based on a receiving radio signal. The circuit diagram for converting digital signal to radio signal is shown in Fig. 5.

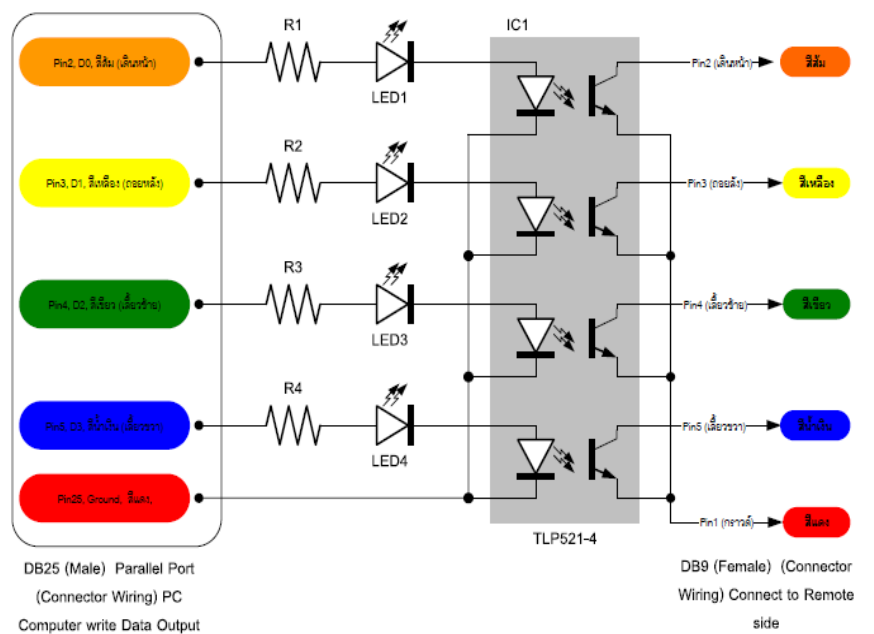

Fig. 5. The circuit diagram for converting digital to radio signal

The digital signal transmits to a parallel port by using the following numbers assignment as shown in Table I.

TABLE I: NUMBER ASSIGNMENT FOR PARALLEL PORT CONTROLLING
\begin{tabular}{|c|l|}
\hline Number assignment & Command \\
\hline $1 \mathrm{H}$ & Right \\
\hline $2 \mathrm{H}$ & Left \\
\hline $4 \mathrm{H}$ & Reverse \\
\hline $5 \mathrm{H}$ & Reverse + Right \\
\hline $6 \mathrm{H}$ & Reverse + Left \\
\hline $8 \mathrm{H}$ & Forward \\
\hline $9 \mathrm{H}$ & Forward + Right \\
\hline A H & Forward + Left \\
\hline $0 \mathrm{H}$ & Stop \\
\hline
\end{tabular}

\section{SySTEM IMPLEMENTATION}

This section explains the system requirement on both hardware and software implementations, which have the following details.

\section{A. System Hardware Implementation}

An RC car is bought with a remote controller from children's toy shop for around 8 US\$. The remote controller communicates with the $\mathrm{RC}$ car with the radio wave range of $40 \mathrm{MHz}$ band. We connect the $\mathrm{RC}$ car remote controller with a digital-to-radio signal converter. After that, we connect the digital-to-radio signal converter with a computer parallel port. The system hardware is shown in the figures below, which has the following details.

- The RC car with the dimension 14.5 X 7.1 X 4.8 Centimeter. The $\mathrm{RC}$ car remote controller as the radio wave range of $40 \mathrm{MHz}$ (as shown in Fig. 6).

- The client and server computers (as shown in Fig. 7)

- A digital-to-radio signal converter with the RS-232 parallel port connection (as shown in Fig. 8).

\section{B. System Software Implementation}

Both client and server computer use the Windows-XP for an operating system. The application software develops based on the Visual Basic 2005.

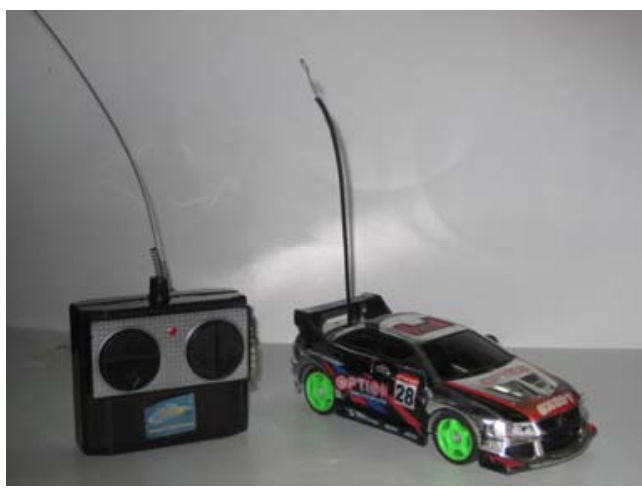

Fig. 6. The RC car with a remote controller.

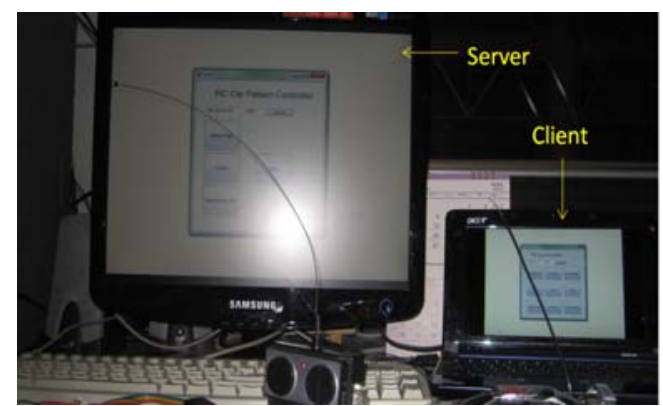

Fig. 7. The client and server computers.

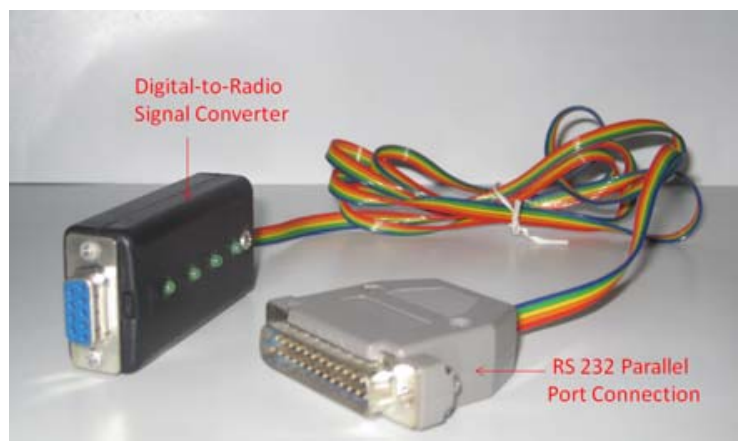

Fig. 8. The analog-to-radio signal converter with the RS-232 paralle connector. 


\section{EXPERIMENTAL RESULTS}

This section presents experimental results of controlling $\mathrm{RC}$ car via the Internet network which is developed and based on the concepts and design mentioned in the previous part. In this research, the experiment's results are focused on the usability and effectiveness of controlling RC car via the Internet network. The experiment conducted on the green carpet size $2 \times 2$ meters. The carpet is an excellent material for simulating a road for an RC car because it has a very good friction between the RC car wheels and carpet's surface.

\section{A. Usability Proof}

In this section, we will analyze the usability of controlling RC car into two parts. First, the user sends the basic commands directly via parallel port. Seconds, the user sends the basic commands between client and server via the Internet network. Finally, the result shows that the usability concept of controlling $\mathrm{RC}$ car via the Internet network can operate in the same way being controlled directly by performing stand alone. The basic commands conducted each command 100 times with the perfect precision rate as shown in Table II.

TABLE II: THE BASIC COMMANDS PRECISION RATE.

\begin{tabular}{|l|l|l|l|}
\hline \multirow{2}{*}{ RC car commands } & \multicolumn{3}{|c|}{ Basic Commands } \\
\cline { 2 - 4 } & No. Testing & No. Success & Precision \\
\hline Forward + Left & 100 & 100 & $100 \%$ \\
\hline Forward & 100 & 100 & $100 \%$ \\
\hline Forward + Right & 100 & 100 & $100 \%$ \\
\hline Left & 100 & 100 & $100 \%$ \\
\hline Stop & 100 & 100 & $100 \%$ \\
\hline Right & 100 & 100 & $100 \%$ \\
\hline Reverse + Left & 100 & 100 & $100 \%$ \\
\hline Reverse & 100 & 100 & $100 \%$ \\
\hline Reverse + Right & 100 & 100 & $100 \%$ \\
\hline \multicolumn{1}{|c|}{ TOTAL } & 900 & 900 & $100 \%$ \\
\hline
\end{tabular}

\section{B. Effectiveness Proof}

The effectiveness test is done the same as that in the previous section, but we will focus on testing three pattern commands. Based on the user commands part, pattern commands are consists of the following patterns.

The O-shape pattern performs the $\mathrm{RC}$ car in the circle-shape pattern (as shown in Fig. 9). The diameter of the circle shape is around 1.5 meters. The experiment considers failure if the RC car cannot run as a circle shape. The experiment conducted 100 times and the precision rate is 97 percent, as shown in Table III.

The $\infty$-shape pattern performs the $\mathrm{RC}$ car in the infinity-shape pattern (as shown in Fig. 10). The diameter of each circles in the infinity shape is around 1 meter. The experiment considers failure if the $\mathrm{RC}$ car cannot run as an infinity shape. The experiment conducted 100 times and the precision rate is 85 percent, as shown in Table III.

TABLE III: THE PATTERN COMMANDS PRECISION RATE.

\begin{tabular}{|l|l|l|l|}
\hline \multirow{2}{*}{\multicolumn{1}{c|}{ RC car commands }} & \multicolumn{3}{c|}{ Pattern Commands } \\
\cline { 2 - 4 } & No. Testing & No. Success & Precision \\
\hline Circle Shape & 100 & 97 & $97 \%$ \\
\hline Infinity Shape & 100 & 85 & $85 \%$ \\
\hline Run \& stop before hit an object & 100 & 97 & $97 \%$ \\
\hline TOTAL & 300 & 279 & $93 \%$ \\
\hline
\end{tabular}

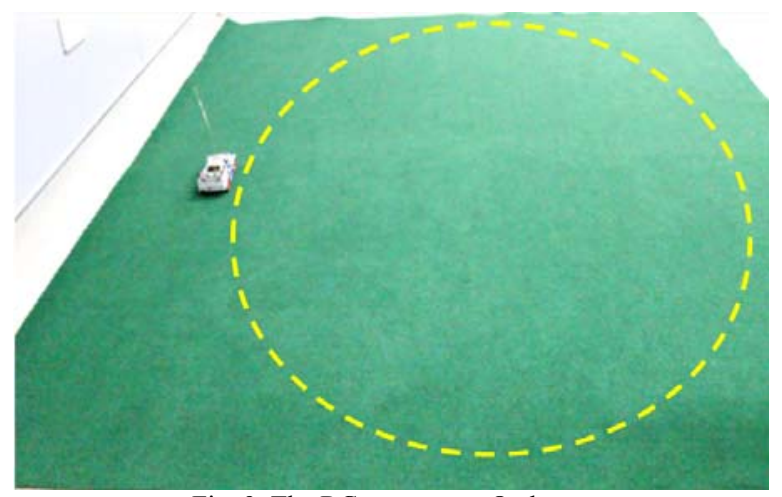

Fig. 9. The RC car runs as O-shape.

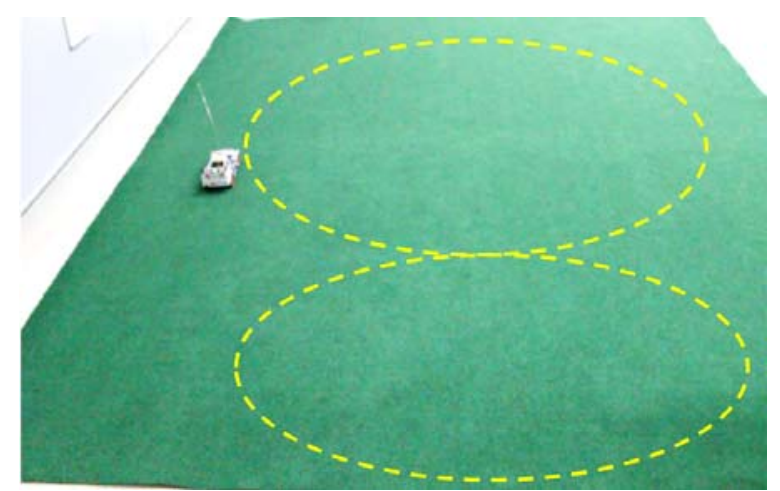

Fig. 10. The RC car runs as $\infty$-shape.

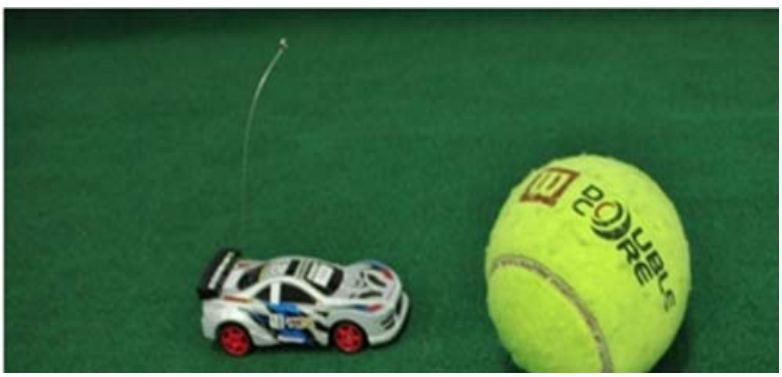

Fig. 11. Run and stop before hit an object operation.

To run \& stop before hit an object performs by running the $\mathrm{RC}$ car in a straight line around 1.5 meters away from the giant tennis ball (as shown in Fig. 11). The experiment considers failure if the $\mathrm{RC}$ car runs to hit the tennis ball or the distance between the $\mathrm{RC}$ car and the tennis ball is more than 30 centimeters. The experiment conducted 100 times and the precision rate is 97 percent, as shown in Table III. The pattern commands conducted totally 300 times and the average precision is 93 percent.

\section{CONCLUSION}

Based on the experimental results in the previous section, the results have fulfilled our research objective. We can conclude that controlling $\mathrm{RC}$ car can be able to perform via the Internet network with basic commands and pattern commands. Nevertheless, the controlling RC car has some limitations as the following.

- The RC car is a cheap car with some hardware performance limitation. It uses a simple motor (not stepper motor) to drive a car. Therefore it will have inertia force after commands were sent.

- The limit distance of radio signal to control an RC car which connects to the server site. So, the car cannot operate far from the server site. 
- The RC car use 1.2 Volts rechargeable battery and need to set to a high level of voltage before perform all testing.

In the future, the TRCC can apply to many applications include: 1) military to detonate a buried bomb, 2) industrial to perform the remote controlling tasks and 3) security guard to monitor the restriction area.

\section{REFERENCES}

[1] Chomtip Pornpanomchai and Phichate Sukklay, "Operating radio-controlled cars by a computer", International Journal of Engineering and Technology (IJET), vol. 3, no. 3, pp. 215-219, June 2011.

[2] Chomtip Pornpanomchai, Phichate Sukklay, and Parichart Leechor "Operation of a radio-controlled car by voice commands", The International Conference on Mechanical and Electronics Engineering, vol.1, pp. 14-17, Kyoto, Japan, 2010.

[3] Chomtip Pornpanomchai, Burin Wiranurak, Woraphol Weerawut, and Linjong Kawrungruang, "Operation of a radio-controlled car using facial commands", The International Conference on Mechanical and Electronics Engineering, vol. 1, pp.108-112, Kyoto, Japan, 2010.

[4] Masanori Yoshinaga, Yukihiro Nakamura, and Einoshin Suzuki, "Mini-Car-Soccer as a testbed for granular computing", The International Conference on Granular Computing, pp. 92-97, Beijing, China, 2005

[5] Christophe Coue and Pierre Bessiere, "Chasing an elusive target with a mobile robot", The International Conference on Intelligent Robots and Systems, pp. 1370-1375, Hawaii, USA, 2001.

[6] Yeung Yam and Kai Man Tong, "A Scaled-Down Testbed for Human Driver Modeling and Evaluation", The International Conference on Robotics, Intelligent Systems and Signal Processing, pp. 376-381, Changsha, China, 2003.

[7] Kenichi Aoto, Masahiro Inoue, Tomoyuki Nagshio, and Takashi Kida, "Nonlinear control experiment of $\mathrm{rc}$ car using internet", The Conference on Control Applications, pp. 1575-1580, Toronto, Canada, 2005.

[8] Chomtip Pornpanomchai and Phichate Sukklay, "Telerobot arm (tra)", The International Conference on Mechanical and Aerospace Engineering, pp. 5161-5166, Bangkok, Thailand, 2011.
[9] Jae-Won. Kim, Byeong Doo Choi, Sang Hee Park, Kyoung Keun Kim, and Sung Jea Ko, "Remote control system using real-time MPEG-4 streaming technology for mobile robot", The International Conference on Consumer Electronics, pp. 200 - 201, USA, 2002.

[10] Dawei W., Jianqiang Y., Dongbin Z., and Guosheng Y., "Teleoperation system of the internet-based omnidirectional mobile robot with a mounted manipulator", The International Conference on Mechatronics and Automation, pp. 1799-1804, Harbin, China, 2007.

[11] Safaric R., Sinjur S., Zalik B., and Parkin R.M., "Control of Robot ARM with Virtual Environment Via the Internet", The Proceedings of the IEEE, Vol. 91 , Issue 3, (2010), pp. 422-429, 2003.

[12] Shuang Cong and Junnan Wang, "Internet-based and visual feedback networked robot arm teleoperation system" The International Conference on Networking, Sensing and Control, pp. 452-457, 2010.

[13] Marin R., Sanz P.J., Nebot P., and Wirz R., "A Multimodal Interface to Control a Robot Arm Via the Web: a Case Study on Remote Programming", The IEEE Transactions on Industrial Electronics,Vol. 52, Issue 6, pp. 1506-1520, 2005.

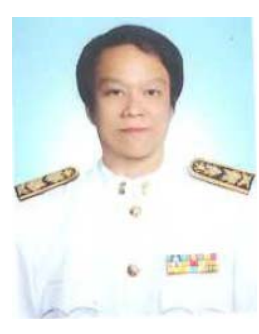

Chomtip Pornpanomchai received his B.S. in general science from Kasetsart University, M.S. in computer science from Chulalongkorn University and Ph.D. in computer science from Asian Institute of Technology. He is currently an assistant professor in the faculty of Information and Communication Technology, Mahidol University, Bangkok, Thailand. His research interests include artificial intelligence, pattern recognition and object-oriented systems.

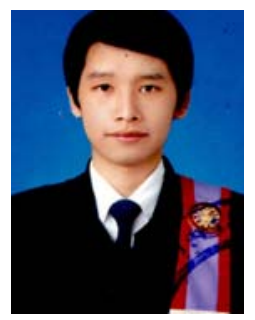

Nutdanai Trakarnsirinont received his B.S. in IT from Thammasat University. $\mathrm{He}$ is currently a master degree student in the faculty of Information and Communication Technology, Mahidol University, Bangkok, Thailand. 